\title{
DIMENSIÓN SOCIAL DE LA TECNOLOGÍA CERÁMICA EN SOCIEDADES CAZADORAS-RECOLECTORAS
}

\begin{abstract}
PALEO, M.C.; PEREZ MERONI, M. Dimensión social de la tecnología cerámica en sociedades cazadoras-recolectoras. Rev. do Museu de Arqueologia e Etnologia, São Paulo, 15-16: 73-85, 2005-2006.
\end{abstract} \\ RESUMO: O objetivo do trabalho é aprofundar o conhecimento sobre a variabilidade \\ morfológica e funcional existente no conjunto cerâmico do sítio Las Marias. A mostra \\ cerâmica procede de um sítio de caçadores-coletores do Holoceno tardio, situado no \\ departamento de Magdalena, Buenos Aires, Argentina. Em um trabalho prévio foram \\ isoladas três categorias de recipientes: processamento, armazenamento e transferência \\ (Paleo e Perez Meroni, no prelo). São analisados em particular os recipientes de \\ processamento. Foi elaborada uma metodologia para abordar a relação forma-função \\ que permitiu compreender a diversidade existente. Os estudos de matéria-prima, \\ evidências de uso e análise de resíduos orgânicos possibilitaram inferir sobre a gama \\ de atividades em que os recipientes haviam sido utilizados. Considera-se que investigar a \\ dimensão social da tecnologia cerâmica permitiu conhecer diferentes estratégias sociais \\ utilizadas por esses grupos.
}

María Clara Paleo*

Mercedes Pérez Meroni*

UNITERMOS: Tecnologia cerâmica - Metodologia - Análise morfo-funcional Processamento - Caçadores-coletores.

\section{Introducción}

Las investigaciones desarrolladas en sitios de cazadores-recolectores del Holoceno tardío, en los cuales la cerámica adopta un rol relevante en los procesos de intensificación económica y social, permitieron abrir una línea de estudio sobre tecnología cerámica específica para estos contextos.

Los estudios se realizan en el noreste de la provincia de Buenos Aires, Argentina; más precisa-

(*) Laboratorio de Análisis Cerámico. Facultad de Ciencias Naturales y Museo. Universidad Nacional de La Plata.Argentina.mcpaleo@fcnym.unlp.edu.ar mmeroni@ciudad.com.ar mente en los partidos de Magdalena y Punta Indio.

Los conjuntos alfareros recuperados se caracterizan por estar compuestos en su totalidad por fragmentos de tamaño pequeño, con alta proporción de tiestos lisos, ausencia de piezas enteras y aparente homogeneidad de los tiestos en manufactura, coloración, tratamiento de superficie y forma.

De la experiencia acumulada se observó que las metodologías utilizadas en forma generalizada para los estudios de cerámica se adecuan a un determinado tipo de muestras y dificultan su aplicación a conjuntos de tipo doméstico.

A lo largo de esta línea de trabajo se ha ido desarrollando una metodología apropiada para el análisis de estos conjuntos, que ha consistido en 
abordar diferentes vías de análisis sobre aspectos tecnológicos, estilísticos, decorativos y de uso. Los estudios se llevaron a cabo mediante la realización de trabajos experimentales (aptitud de los barros, replicación); aplicación de técnicas arqueométricas (Rx, cortes delgados, microscopio electrónico, porosidad, análisis de residuos orgánicos) y análisis morfo-funcional (Balesta et al. 1997; Pérez Meroni y Blasi 1999; Paleo y Pérez Meroni 1995; 1999; Paleo y Pérez Meroni (1) en prensa).

Se han aislado, hasta el momento, tres categorías de contenedores: procesamiento, almacenaje y transferencia propuestos en un trabajo previo (Paleo y Pérez Meroni (2) en prensa).

El objetivo del presente trabajo es profundizar el conocimiento sobre la variabilidad morfológica y funcional existente en el conjunto alfarero del sitio Las Marías. La muestra seleccionada procede de un sitio de cazadores-recolectores y pescadores del Holoceno tardío, ubicado en el partido de Magdalena, provincia de Buenos Aires, Argentina. Para la finalidad propuesta se analizan en particular los contenedores de procesamiento.

Explorar aspectos de esta tecnología en sociedades cazadoras-recolectoras constituye un aporte para lograr mayor información sobre estrategias de movilidad, comunicación e intercambio, subsistencia, prácticas domésticas, entre otras.

\section{Caracterización de las ocupaciones humanas del área de estudio}

En la franja costera de los partidos de Magdalena y Punta Indio en la provincia de Buenos Aires, que corresponde al litoral del Río de la Plata, se han estudiado siete sitios de los doce identificados hasta el momento. Seis de ellos conforman la localidad arqueológica Barrio San Clemente en el partido de Punta Indio y el otro denominado Las Marías en el partido de Magdalena. Estos sitios comparten una serie de características ambientales y culturales.

En el área de estudio, existe una correspondencia entre las diferentes topografías, tipos de suelos y las formaciones vegetales que desarrollan a lo largo del gradiente continente - costa del Río de la Plata. Desde el interior hasta la costa se reconocen tres zonas que a continuación se detallan:

La Llanura alta, presenta un relieve suavemente ondulado con cotas mayores de $5 \mathrm{~m}$, donde predominan suelos loessoides e intergrada con depresiones de suelos arcillo-limosos. En la misma se desarrolla la estepa graminosa (flechillar), que por su composición florística y extensión regional es considerada una formación climáxica. En las áreas de depresiones están presentes las praderas húmedas.

El paisaje se continúa con la Llanura de cordones de playa, que comprende un conjunto de acumulaciones cordoniformes, conglomerádicas, conchiles y arenosas subparalelas entre sí, se suceden en niveles decrecientes y se escalonan hacia la costa. En el área de estudio, estos depósitos marinos corresponden a la Formación Las Escobas, Facie Cerro la Gloria (Fidalgo et al. 1973). Según las discontinuidades, granulometría y la altura de los bancos, se han diferenciado cuatro sistema de bancos (I, II, III, IV) separados por depresiones intercordonales (Cavallotto 1995). El sistema I es el más antiguo, se encuentra hacia el interior con cotas de $5 \mathrm{~m}$. Los sitios arqueológicos estudiados se ubican en el sistema III con cotas de 2-2,5 m, aproximadamente a 1-1.500 km de la costa. En los suelos calcáreos de los cordones de conchilla se desarrolla el bosque xerófilo conocido como "bosque de tala", conformado por ocho especies arbóreas: Celtis tala (tala), Scutia buxifolia (coronillo), Jodina rhombifolia (sombra de toro), Schinus longifolius (molle), Sambucus australis (sauco), Phytolacca dioica (ombú), Sapium hacmatospermum (curupí) y Colletia spinosissima (brusquilla). Así también, la cobertura de las especies herbáceas, trepadoras y rastreras varía según la proximidad del cordón con respecto a la planicie de inundación. Los árboles introducidos forman bosquecillos densos muy próximos y/ o que penetran en el talar.

Por último, entre la Llanura de cordones de playa y la costa se desarrolla la Planicie de inundación, ambiente sometido a inundaciones periódicas. Alo largo de la misma, en suelos poco desarrollados del tipo hidromórfico-halofíticos, predominan comunidades vegetales edáficas, comunidades hidrófilas (juncal, totoral, vegas de Cyperaceas) y halófilas (pradera salada, espartillar); próximo a los numerosos cauces que surcan el área, se desarrollan comunidades hidrófilas (pajonales de cortaderas, vegas).

Asimismo, este paisaje se encuadra en un ambiente fluvial, definido por la presencia de los ríos Samborombón, Río de la Plata y tributarios menores. 
PALEO, M.C.; PEREZ MERONI, M. Dimensión social de la tecnología cerámica en sociedades cazadoras-recolectoras. Rev. do Museu de Arqueologia e Etnologia, São Paulo, 15-16: 73-85, 2005-2006.

Los sitios arqueológicos estudiados se encuentran localizados en la zona denominada Llanura de cordones de playa cuya secuencia sedimentaria se caracteriza, en todos los casos, por un sustrato II CK, constituido por gravas calcareníticas con intercalaciones de niveles arenosos. Sobre este depósito marino se ha depositado un suelo inmaduro de aproximadamente $0,60 \mathrm{~m}$ de profundidad, que no ha alcanzado una diferenciación marcada de horizontes. Este suelo comprende un horizonte A de 0,30 m muy rico en materia orgánica, en complejos húmicos y contenido de carbonato de calcio y $\mathrm{pH}$ alcalino. El horizonte A pasa gradualmente hacia un horizonte AC, de 0,30 m de potencia media, caracterizado por un elevado contenido de carbonato de calcio y menor de materia orgánica. Este suelo es la resultante de una dinámica importante caracterizada por la permanente incorporación, transformación y pérdida de material orgánico y clástico al perfil del suelo.

El material arqueológico se localiza en la parte superior de la secuencia sedimentaria, observándose la mayor concentración del material entre los $0,25 \mathrm{~m}$ - 0,40 m. En líneas generales, el registro arqueológico de los sitios mencionados está conformado por abundantes fragmentos de cerámica, restos óseos humanos y faunísticos, instrumental óseo, material e instrumental lítico en escasa representatividady estructuras de combustión. Son sitios a cielo abierto, y el análisis contextual ha permitido definirlos como unicomponentes y de actividades múltiples, sin identificar hasta el momento sectorización de actividades.

Se ha postulado la presencia del bosque de tala en los momentos de ocupación de los sitios y se ha corroborado mediante la realización de estudios palinológicos y ambientales (Paleo et al. 2000). Los resultados obtenidos han sido analizados conjuntamente con información etnobotánica y etnohistórica y han permitido inferir la utilización de los recursos del bosque, a través de la recolección, como componentes importantes de la subsistencia.

El análisis del registro arqueofaunístico evidencia que mayoritariamente está representado por cérvidos, roedores y peces. En escasa representatividad se identifican restos de Lama guanicoe (guanaco). El análisis de los recursos utilizados en la subsistencia ha tenido en cuenta para cada uno de ellos la abundancia relativa en cada sitio, el aporte a la dieta y la procedencia.
La caza estuvo orientada fundamentalmente a recursos autóctonos como los cérvidos que constituyen el recurso que más ha aportado a la dieta y también ha incidido en el subsistema tecnológico evidenciado en la utilización de huesos y astas como materia prima para la fabricación de punzones, agujas y perforadores. Se registran dos tipos de cérvidos: Blastoceros dichotomus (ciervo de los pantanos) y Ozotoceros bezoarticus (venado de las pampas).

También han formado parte de la dieta con un aporte significativo los roedores de gran tamaño presentes en todos los sitios, tales como Myocastor coipus (coipo), Lagostomus maximunn (vizcacha) y Hydrochaeris hydrochaeris (carpincho).

Así también se observa el aprovechamiento de recursos variados como Tupinambis sp.(lagarto overo) y Dasypus sp.(mulita).

Otro recurso muy abundante y con mayor rendimiento en la provisión de carne es el taxón peces especialmente Pogonias cromis (corvina negra) y armados de gran tamaño (Pimelodidae y Doradidae.) Se destaca la utilización de huesos pterigióforos de la aleta anal para la confección de instrumentos formatizados con evidencias de uso. La cantidad de restos recuperados sumado al grado de predictibilidad y abundancia de este recurso, ha permitido caracterizar a estos grupos como pescadores.

La caza del guanaco es interpretada como ocasional en relación a la escasez de restos recuperados y se lo considera como un recurso alóctono dado que el bosque de tala no es su hábitat preferencial. La presencia en el sitio mayoritariamente de partes del esqueleto apendicular, podría deberse a un trozamiento en el lugar de matanza y su posterior traslado.

A partir de este análisis se propone que las estrategias de subsistencia han puesto énfasis en la caza, pesca y recolección.

El registro tecnológico de estos sitios se caracteriza por la presencia de abundante material cerámico, permitiendo considerar a estos grupos como ceramistas. La tecnología cerámica constituye una innovación tecnológica importante y existe consenso acerca de su emergencia en la región pampeana en contextos de cazadores-recolectores en algún momento del Holoceno tardío. Así también y sobre la base de estudios etnoarqueológicos, la alfarería forma parte de la cultura material de grupos móviles. En los cazadores-relecolectores de 
la región pampeana su incorporación constituyó un avance importante dentro de su tecnología, como así también significó un cambio en aspectos económicos y simbólicos (Politis, Madrid 2001). El registro también está compuesto por instrumental óseo relativamente abundante, y escaso instrumental lítico. Es importante destacar que en la zona no se registra la presencia de fuentes de aprovisionamiento de materia prima lítica (cuarcita, cuarzo cristalino y calcedonia) localizándose las más próximas unos 300 km hacia el SO.

Hasta el momento y a modo de síntesis, se ha podido interpretar el modo de subsistencia de estos grupos como cazador-recolector y pescador, evidenciando un uso intensivo de los recursos locales, tanto para la subsistencia como para la tecnología cerámica y ósea, con una baja movilidad residencial. La escasa presencia de recursos alóctonos, como Lama guanicoe y la materia prima lítica, en los asentamientos ha permitido inferir que estos grupos intervendrían en redes de intercambio e interacción de escala regional.

En la actualidad se dispone de nueve dataciones radiocarbónicas para el área de estudio, la interpretación conjunta de los registros arqueológico, cronológico y palinológico ha permitido proponer la ocupación del área desde ca.1600 años.

Esta caracterización de las ocupaciones durante el Holoceno tardío es compartida por otros sitios del área norte de la provincia de Buenos Aires y de la Depresión del Salado (Politis 1988).

\section{Metodología}

Como se ha mencionado el objetivo del trabajo propone profundizar el conocimiento sobre la variabilidad morfológica y funcional existente en un conjunto alfarero y en particular el análisis de los contenedores de procesamiento.

Para tal fin se ha seleccionado el material cerámico del sitio Las Marías. Este sitio, con una superficie excavada de $40 \mathrm{~m}^{2}$, presenta un registro arqueológico que no difiere de los otros estudiados en el área. Se cuenta con dos dataciones realizadas en el INSTAAR Laboratory for AMS Radiocarbon Preparation and Research, University of Colorado at Boulder. Las muestras seleccionadas, que proceden de la misma unidad estratigráfica, corresponden a restos óseos de Pogonias cromis y de Lama guanicoe. Las dataciones obtenidas han sido de $1820 \pm 50$ años APy $1590 \pm 40$ años AP, respectivamente. Efectuado el efecto reservorio al fechado de Pogonias cromis, se ha establecido la contemporaneidad de ambas muestras y ubicado cronológicamente la ocupación del sitio en ca. 1500-1600 años AP.

El conjunto cerámico del sitio está compuesto por 4932 fragmentos y 16 masas de arcilla sin cocinar, posee un alto grado de fragmentación, los tiestos en una alta proporción son lisos con aparente homogeneidad en la manufactura, coloración, tratamiento de superficie y forma. Cabe aclarar que no se dispone de piezas enteras que sirvan como colección de referencia.

Se le asigna al conjunto un uso generalizado de tipo doméstico y en tal sentido las vasijas han sido consideradas contenedores. De este modo, se ha tomado la clasificación de categorías de uso, propuesta por Rice que agrupa los contenedores en clases de procesamiento o transformación, almacenaje y transferencia o transporte (Rice 1989).

Para poder aplicar esta clasificación ha sido necesario introducir una nueva variable en el análisis del conjunto como es la forma del contenedor, se considera que la misma debe ser abordada conjuntamente con las categorías de uso, estableciendo un análisis de la relación forma-función. La forma y el uso de la vasija se encuentran íntimamente relacionadas y determinadas por ideas normativas, modas y aspectos tecnológicos. Si bien el uso de una vasija puede cambiar a través de su vida, se espera que la forma de la pieza esté ligada al uso y que se puedan establecer relaciones generales entre la forma de la vasija y su función primaria (Sinópoli 1991). Como propuesta se ha elaborado una metodología para abordar la relación forma-función en estos conjuntos. Las categorías morfo-funcionales aplicadas a conjuntos cerámicos fragmentarios procedentes de contextos de cazadores-recolectores, han permitido comprender la diversidad existente, a pesar de su aparente homogeneidad.

Como punto de partida, la revisión bibliográfica realizada, sobre el tratamiento y clasificación de formas, evidencia la necesidad de adecuar las propuestas a este tipo de muestras (Shepard 1976; Balfet et al. 1992; Orton et al. 1997; Rice 1989). El principal inconveniente radica en que estas clasificaciones han sido elaboradas para conjuntos cerámicos de piezas completas y/o para conjuntos fragmentarios comparables con colecciones de referencia. Es así, que las clasificaciones más utilizadas se basan en contornos y proporciones de las vasijas, u otras, donde se utilizan medidas tales como altura máxima, diámetro máximo y mínimo. 
En la muestra cerámica utilizada en este trabajo, no se pueden relevar la mayoría de los parámetros requeridos por estas clasificaciones, algunos se infieren en forma indirecta como por ejemplo el diámetro de la boca, y otros se estiman por cálculos matemáticos, de este modo la descripción de las formas puede expresarse como "una tendencia a ..." formas globulares, hemiesféricas, entre otras. Estas categorías basadas en tendencias han sido útiles como marco descriptivo, pero para avanzar en el marco predictivo y poder establecer al menos que un grupo de vasijas que parecen semejantes puedan haber sido usadas de una determinada manera, se necesita adicionarle la variable funcional. $\mathrm{Al}$ abordar este tipo de estudio se reconoce, como comenta Skibo que el término función es más amplio y abarcativo que la utilización del artefacto en sí mismo debido que incluye también aspectos sociales e ideológicos. En esta línea de trabajo los estudios tecno-funcionales constituyen el punto de partida para analizar la interrelación con la función social e ideológica del artefacto (Skibo 1992).

Como el objetivo de este trabajo es ampliar la información sobre los contenedores de procesamiento, a modo de síntesis y en función a trabajos previos (Paleo y Pérez Meroni (2) en prensa) se comentan las características de las otras categorías.

Contenedores para almacenaje: se han asignado a esta clase 52 fragmentos. Estas formas se caracterizan por presentar bocas con aberturas pequeñas, con diámetros que oscilan entre 3 y $6 \mathrm{~cm}$ y paredes muy gruesas con espesores entre 1 y 2,5 cm y cuellos con marcados puntos de inflexión próximos a bordes evertidos. Poseen un buen acabado de superficie con baño de pintura blanca en ambas caras (Foto 1).

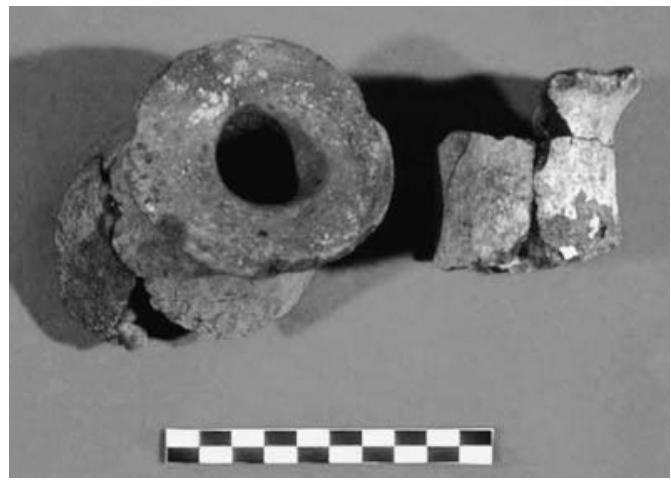

Foto 1 - Contenedores de almacenaje.
Son interesantes las observaciones que se han realizado a partir de los resultados de las difractometrías por Rx sobre la capa de pintura, en cuya composición se destaca la presencia de zeolitas (analcima) que posee la propiedad de absorción de olores.

Estos contenedores no se han podido reconstruir en su totalidad. El análisis bibliográfico de la arqueología regional permite contrastar la ausencia de formas completas. En tal sentido, Ameghino en su publicación de 1880, ilustra y describe estas formas como botellones. Así también Cigliano (1963) hace mención a estas formas particulares para la región de Punta Indio.

Se estima que podrían tener un uso vinculado al almacenaje de líquidos o de sustancias secas y procesadas.

Contenedores de transferencia: se han asignado a este grupo 108 fragmentos; algunas piezas han podido ser reconstruidas hasta un $60 \%$.

Estos objetos presentan dos extremos abiertos, uno pequeño entre 2 y $4 \mathrm{~cm}$ que se prolonga a manera de tubo ensanchándose en el otro extremo con una abertura variable entre 7 y $10 \mathrm{~cm}$ de diámetro y con una longitud promedio de $15 \mathrm{~cm}$. Se han relevado algunos fragmentos con decoración incisa (Foto 2).

La forma particular de estos objetos, con un extremo tal vez para verter supondría una función en el traspaso de sustancias tanto líquidas como sólidas. A partir del análisis de la pasta se desprende el origen alóctono de la materia prima.

Dada la peculiaridad de estos objetos se ha realizado un relevamiento bibliográfico sobre la presencia de los mismos en sitios arqueológicos de la región. De la misma se desprende que no existe un consenso acerca de su denominación y uso, existiendo variadas interpretaciones desde las primeras investigaciones de Moreno (1874) en la región.

\section{Contenedores de procesamiento}

Del total de la muestra los fragmentos asignados a contenedores de procesamiento es de 1156 tiestos, de los cuales se han remontado 598 que corresponden al $12 \%$ de la muestra, en algunos casos se ha podido reconstruir hasta el $50 \%$ de la pieza. No se han contabilizado los bordes menores a $2 \mathrm{~cm}$.

La idoneidad de una vasija para realizar ciertas funciones puede obtenerse a partir del estudio de su 


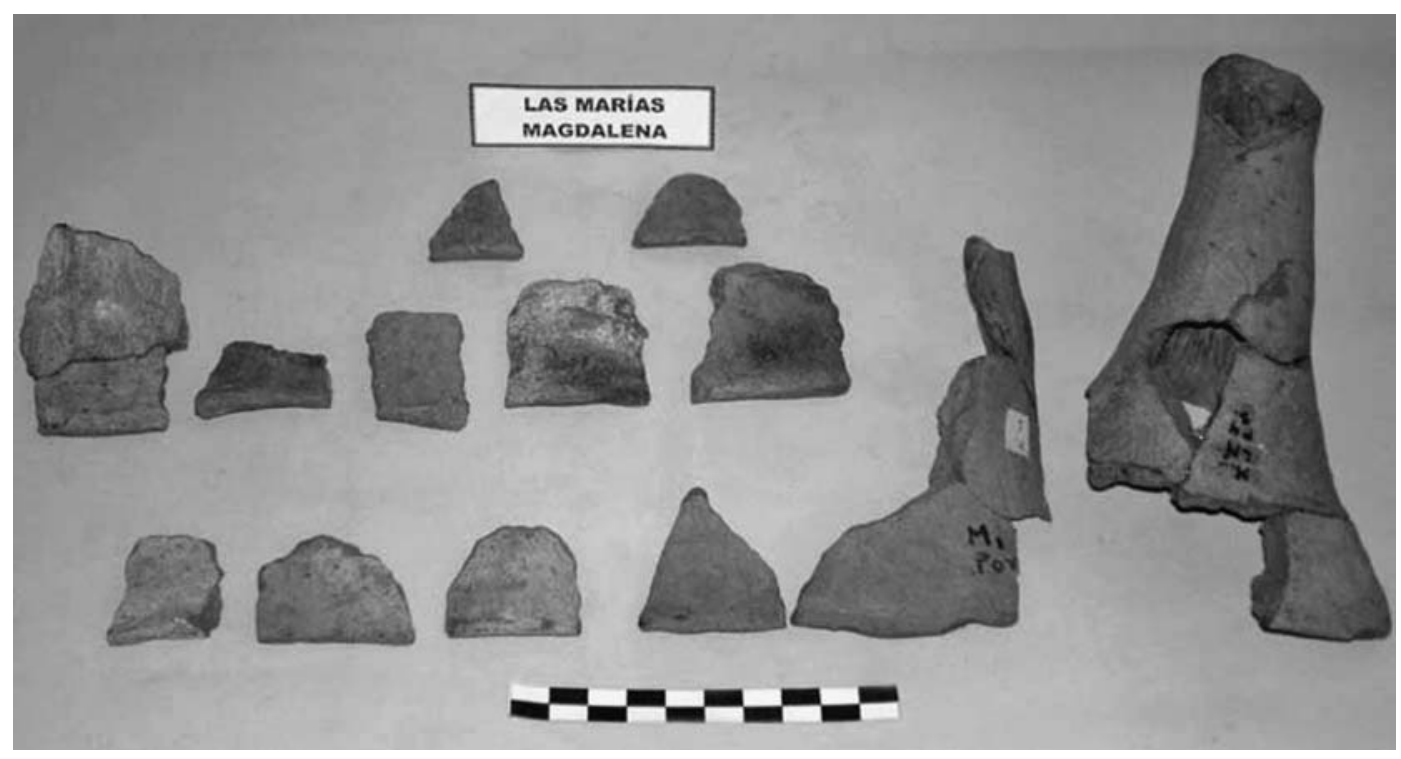

Foto 2 - Contenedores de transferencia.

forma y características físicas. Algunos autores proponen que para realizar una aproximación a la relación forma y función de las vasijas, se debe conocer las características físicas de la arcilla cocida que brinda información sobre el uso final del producto (Orton 1997: 248).

Se propone para estos contenedores de procesamiento un uso vinculado a las actividades de preparación y cocción de alimentos. Es así que se utilizan para el análisis morfo-funcional variables tales como la forma, la materia prima, evidencias de uso y análisis de residuos orgánicos (Skibo, 1992).

\section{Análisis de la forma}

Se considera importante destacar algunos factores que se han tenido en cuenta en este análisis. Se parte de la premisa que no se conoce el tamaño original de la muestra sumado a que se analiza una muestra de tipo fragmentaria. Otro factor adicional es la relación entre el número de tiestos recuperados de la excavación y la cantidad real de contenedores que ellos representan.

También se debe estimar el diferente grado de rotura de los distintos tipos de contenedores en su etapa de uso (Sinópoli 1992:87).

Como punto de partida se comenzó con el remontaje de fragmentos, que ha resultado de importancia para la reconstrucción de piezas. El inicio de esta actividad consistió en el seguimiento de los bordes, brindando una importante fuente de información. Los tiestos del cuerpo resultan más difíciles de remontar, y algunas veces se dificulta su ubicación en la pieza. En otros casos, existen marcadas diferencias en los grosores de las paredes y/o presencia de líneas de pulido que ayudan a su ubicación y orientación. Conjuntamente con esta tarea se realiza el seguimiento de fragmentos por niveles y sectores registrando la migración de los mismos.

Se han podido identificar algunas formas que hasta el momento no se tenían registradas, introduciendo el concepto de variabilidad en el conjunto. Se considera que toda tipología, tiene que brindar la posibilidad de poder expandirse, al recuperar más material de las excavaciones que introduzca variabilidad o por la aplicación de nuevos estudios que permitan una mayor especificación.

Los contenedores de procesamiento han sido agrupados de acuerdo al diámetro de boca en: pequeños: 8 - $12 \mathrm{~cm}$ (7,5\%), medianos: 16 - $28 \mathrm{~cm}$ (64,2 \%) y grandes: $32-50 \mathrm{~cm}(28,3 \%)$.

En nuestro conjunto el número mínimo de contenedores se obtiene agrupando los fragmentos remontados y aquellos que si bien no remontan se asignan a la misma pieza. Las variables tenidas en cuenta para tal fin se evalúan en forma combinada: características de la pasta, acabado y coloración de 
la superficie interna y externa, decoración, evidencias de uso como presencia de hollín, manchas de ahumado, marcas, marcas de cocción, grosor de paredes, curvatura de los fragmentos y puntos de inflexión, medición del diámetro de la boca. Esta última variable resulta muy útil para realizar un primer agrupamiento y por otro lado esta medición depende de una parte de la vasija que podemos asignar con seguridad. Es así que a partir de las características analizadas se ha podido estimar un Número Mínimo de Contenedores de 53 agrupadas de la siguiente manera: 3 pequeñas, 32 medianas y 18 grandes. $\mathrm{Al}$ analizar conjuntamente el Número Mínimo de Contenedores con los diámetros de las bocas se puede apreciar que la mayor cantidad de contenedores corresponden a recipientes con aberturas amplias $(24 \mathrm{~cm}, 28 \mathrm{~cm}$ y $32 \mathrm{~cm}$ ) propicias para un buen acceso a los contenidos (Tabla 1).

En líneas generales se ha caracterizado la forma de estos contenedores como globulares o subglobulares, bases cóncavas, bocas amplias y ausencia de apéndices a manera de asas. Se han identificado agujeros de suspensión en baja cantidad, los que han sido realizados de adentro hacia fuera.
Del análisis de estas formas se han podido estimar dos tendencias, una de contornos simples esféricos o con pequeños puntos de inflexión próximos al labio con bases cóncavas que se continúan en el cuerpo, donde el diámetro mayor se encuentra en el cuerpo y supera la altura. La otra corresponde a formas donde el diámetro máximo se corresponde con la boca que es igual al diámetro del cuerpo con bases cóncavas (Fotos 3 y 4).

En cuanto a la decoración el área a decorar es siempre la parte superior de la pieza, acotada al labio y borde, abarca aproximadamente de 4-6 cm, las unidades mínimas identificadas son líneas (rectas, quebradas, onduladas) y puntos (variedad de incisiones de acuerdo a los instrumentos utilizados, que se expresan en una gran variedad de trazos).

De la combinación de las unidades mínimas y de los trabajos de replicación y experimentación se han identificado tres tipos de unidades cognitivas que manifiestan la concepción utilizada para definir el espacio a decorar. Una, conformada por bandas horizontales paralelas al labio contiguas en sentido vertical, en estas bandas se aplican la combinación de las unidades mínimas. La otra, se expresa mediante la delimitación de campos por líneas

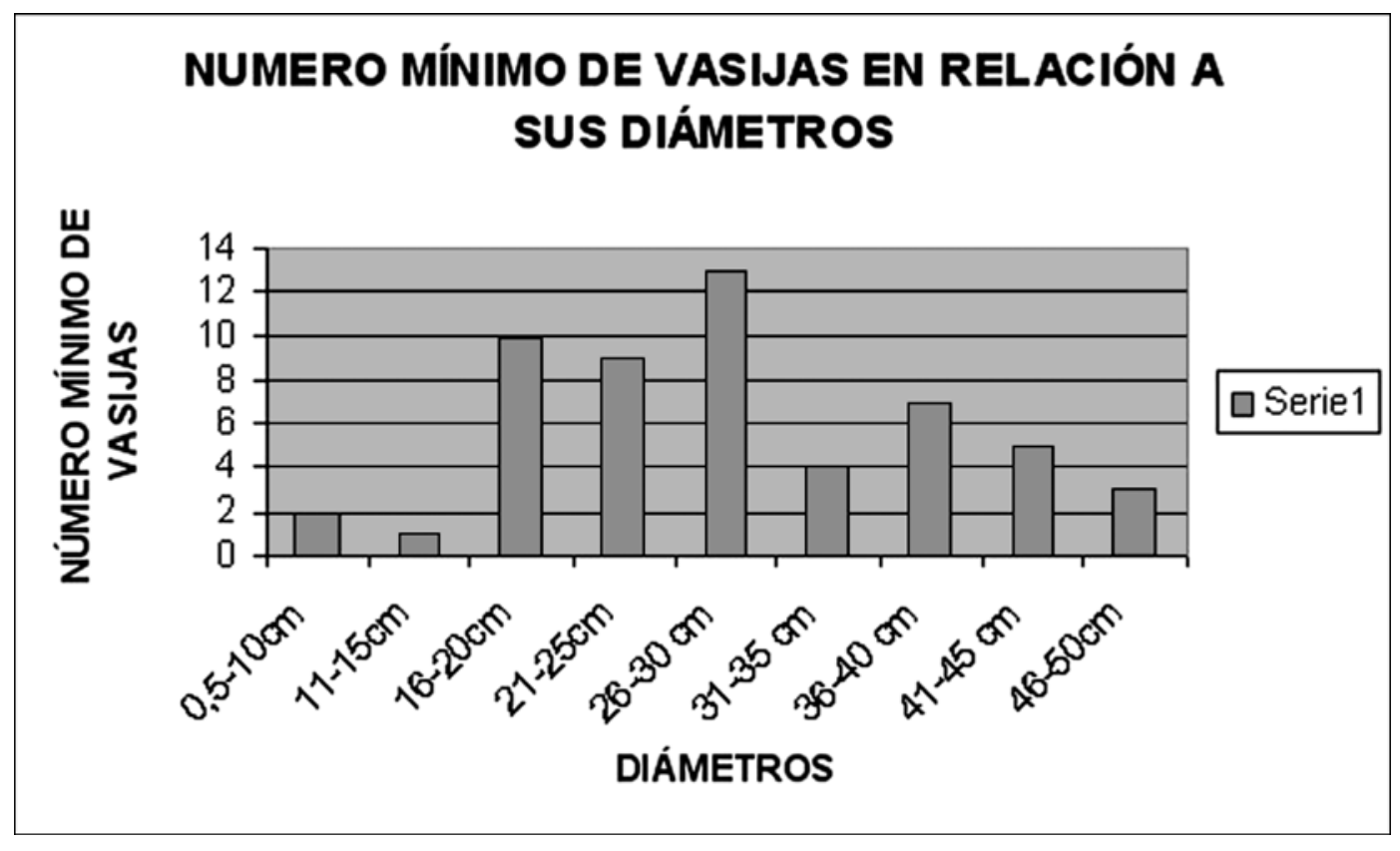

Tabla 1: Número Mínimo de contenedores. 

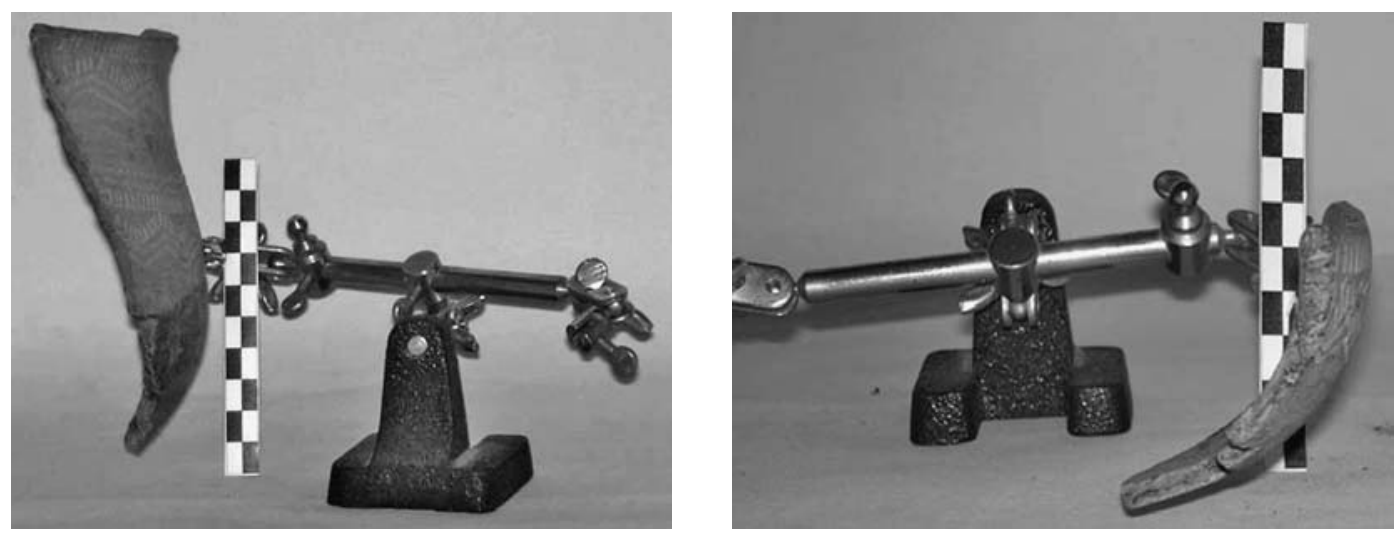

Fotos 3 y 4 - Formas estimadas de los contenedores de procesamiento.

rectas combinadas con líneas quebradas en los que se configuran, mediante la incisión, juegos de figura fondo. La tercera, corresponde a la alternancia de sectores de bandas paralelas con, sectores de delimitación de campos, en la misma pieza. Los motivos están confeccionados por incisión, pintura y combinación de ambas técnicas.

Los estudios sobre decoración parten de considerar al estilo como un sistema abierto de expresión que recibe y transmite información acerca de la identidad de la sociedad que lo produce y acerca de la situación o localización en que aparece (Paleo y Pérez Meroni 1995), este marco de referencia acuerda con la propuesta de Rice que apunta a una articulación de lo constructivo con lo artístico a través del concepto de diseño y de la adecuación de este al uso. Siguiendo estos lineamientos los sectores decorados son concebidos también como una superficie antideslizante que favorece la toma de la pieza.

El análisis de los aspectos funcionales de la forma ha permitido observar que las bases cóncavas son más ventajosas para la cocción debido que transmiten el calor más fácilmente y son más resistentes a la rotura por stress térmico que las bases planas. Otra ventaja que presentan es la facilidad para acceder y volcar su contenido. Estas bases permiten una mayor circulación de aire en la fuente de calor mejorando la combustión.

\section{Análisis de las materias primas}

Se realizaron estudios de la pasta con lupa binocular, composicionales por difractometría de Rx, de porosidad y evaluación del stress térmico.
La selección de las muestras se organizó partiendo de los tres grupos de contenedores definidos como pequeños, medianos y grandes.

Para el grupo de tamaño grande se seleccionaron tres muestras de fragmentos de boca y/o próximos y tres fragmentos asignados a bases o próximos por la presencia de parches de carbón como evidencias de uso. También se seleccionaron tres muestras de fragmentos próximos a la boca de piezas medianas $\mathrm{y}$ tres correspondientes a pequeñas.

A partir del análisis con lupa binocular se identificaron en la pasta granos individuales de cuarzo y feldespato de tamaño pequeño y abundante cantidad de material negro sin determinar a manera de pátina. La matriz es de color ocre y se clasificó como limo-arcillosa. Se han reconocido inclusiones intencionales de tiesto molido, el tamaño del mismo oscila entre 0,5-3,0 mm, con un porcentaje de aproximadamente $5 \%$. Esta incorporación resulta interesante debido que las partículas tienen las mismas propiedades de expansión termal que la pasta cruda, y sugerirían que no están trabajados por un neófito (Meherer 1998:139). En este caso otorgaría la propiedad de minimizar el stress térmico y actuaría como material fundente al bajar el punto de fusión produciendo una cocción más rápida.

Los estudios por difracción de Rx se realizaron en muestra total y fracción arcilla. En la lectura de los difractogramas se han identificado feldespato potásico y plagioclasas (feldespatos sódicos y cálcicos) cuarzo y arcilla de tipo illita. De la relación entre sus proporciones se observa una mayor proporción de feldespato sobre cuarzo cristalino y en menor proporción arcilla de tipo illita siendo esta 
la predominante dentro de las arcillas. Se han realizado algunas observaciones sobre la composición de la pasta tales como la presencia de feldespato, que actúa como material fundente, tiene la propiedad de bajar el punto de fusión produciendo una cocción más rápida y en consecuencia un ahorro de combustible. También la individualización de granos de cuarzo, material refractario, tiene la propiedad de absorber y conservar el calor evitando la rotura o rajadura de las piezas. Esta evaluación ha permitido considerar la aptitud de la pasta por su respuesta al stress térmico, e inferir la utilización de estos contenedores para su exposición al fuego y actividades de procesamiento y transformación de alimentos.

Los estudios realizados sobre porosidad de la pasta se realizaron sometiendo la muestra a hervido y secado. Para tal fin se muestrearon 3 fragmentos de distintas partes del cuerpo de los tres tamaños de contenedores. Los porcentajes obtenidos indican que todas las pastas presentan un porcentaje de porosidad de $35,5 \%$. Si bien la pasta es considerada porosa, todas las vasijas presentan buen acabado de superficie por alisado y otras, por aplicación de engobe o pintura, otorgando a la paredes de los recipientes cierta impermeabilidad. Por este motivo este tipo de estudios debe interpretarse en relación a otras variables tecnológicas, es así que si la pasta tiene poros grandes se producirán menos fracturas debido a la interrupción de la fractura por el poro.

\section{Evidencias de uso}

El conjunto cerámico presenta un total de 558 fragmentos con evidencias de uso. Las mismas consisten en rasgos de distinto tipo que permiten reforzar la función culinaria de los contenedores. De la muestra se han contabilizado 214 fragmentos con adherencias o parches de carbón en la cara interna, con hollín en la cara externa 235 y con adherencias en cara interna y hollín en la externa 109 fragmentos (Foto 5).

Es importante evaluar las variaciones en la cobertura de hollín que se producen de acuerdo a las condiciones y ubicación de la fuente de calor.

La observación de horneado de piezas cerámicas experimentales en hornos abiertos con leña de la zona, y la observación de los fragmentos arqueológicos ha permitido contrastar que la ubicación de los contenedores para la cocción de alimentos ha sido en algunos casos directa sobre el

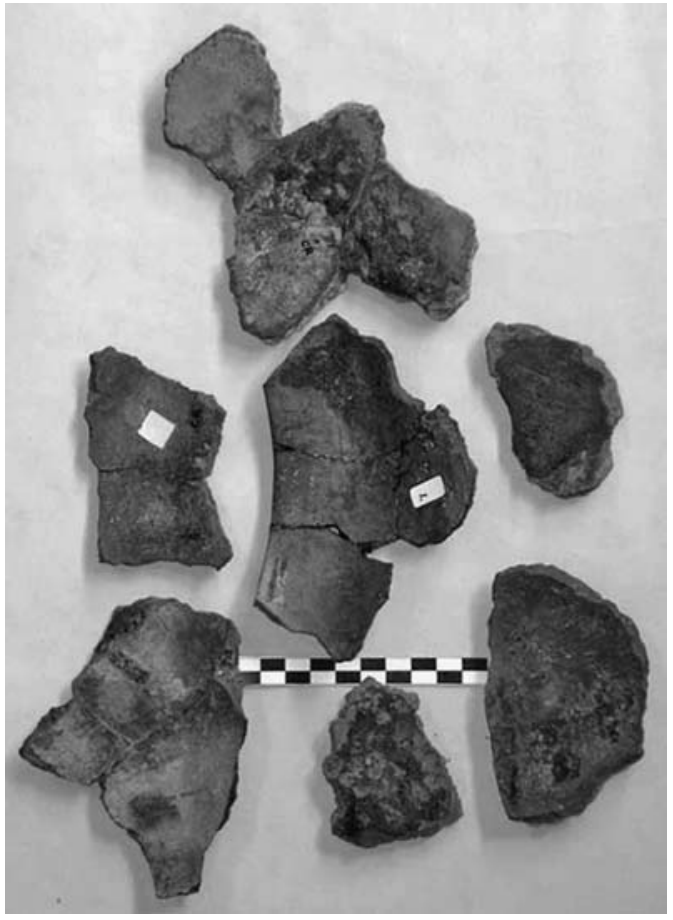

Foto 5 - Evidencias de uso: parches de carbón.

fuego. Esta consideración se fundamenta en que los fragmentos asignados a la base presentan la superficie externa sin evidencias de hollín y las superficies internas quemadas y con adherencias. Así también, se infiere que algunas piezas han sido ubicadas a un costado del fuego en el proceso de cocina dejando en un mismo contenedor partes con hollín y otro sector sin evidencias de ahumado.

\section{Absorción de residuos}

Con el fin de obtener mayor información relacionada con aspectos de la dieta, prácticas de almacenamiento y procesamiento de alimentos, se implementaron análisis químicos sobre restos orgánicos en tiestos cerámicos. Esta metodología permite abordar problemas vinculados con la dieta de grupos pasados desde otra perspectiva como es el almacenamiento y cocción de los alimentos (Paleo y Pérez Meroni, en prensa 1).

Si bien los resultados detallados de este estudio fueron presentados en un trabajo previo (Paleo y Pérez Meroni, op cit) se realizan algunos comentarios sobre su implementación con la finalidad de interpretarlos conjuntamente. 
PALEO, M.C.; PEREZ MERONI, M. Dimensión social de la tecnología cerámica en sociedades cazadoras-recolectoras. Rev. do Museu de Arqueologia e Etnologia, São Paulo, 15-16: 73-85, 2005-2006.

El análisis químico de los restos orgánicos provee registros de los diferentes ácidos grasos que aparecen como residuos visibles en la cerámica o absorbidos por las paredes de los recipientes de cocción y almacenaje. En este caso en particular algunos tiestos presentan adherencias conservadas de restos orgánicos quemados en su cara interna conformando una capa con un espesor medio de 3 $\mathrm{mm}$. Como paso previo al análisis de residuos orgánicos se aplicó la técnica de infrarrojo para determinar si las adherencias eran o no de materia orgánica. Este tipo de análisis es rápido y económico.

Dicho estudio se ha realizado en el Department of Anthropology, University of California, Santa Bárbara, en el "Laboratorio de análisis de restos orgánicos en cerámica” a cargo de el Dr. Stuart T. Smith.

Los resultados obtenidos indican que en todas las muestras procesadas fueron identificados tipos similares de ácidos grasos, esto permitiría inferir que en los recipientes de donde proceden los fragmentos se cocinó el mismo alimento.

En la lectura de los resultados se identificaron ácido palmítico como el más abundante entre las grasas saturadas y, en menor proporción, pero en cantidades aún sustanciales, el ácido esteárico. Las grasas insaturadas identificadas fueron ácido oléico y linoléico. También se identificaron pequeñas cantidades de ácido mirístico. En el procesamiento de los resultados se utilizó el test de envejecimiento rápido elaborado por Malainey (1997), a través del cual se simula la descomposición durante un extenso período de tiempo de los restos orgánicos. Malainey ha establecido, que este resultado donde la relación entre las proporciones es de alto porcentaje de ácido palmítico, moderado de ácido esteárico, oleíco y linoléico, y bajo de ácido mirístico es típica tanto de peces como de maíz dulce. Según Smith se podría descartar la posibilidad que los peces y el maíz hayan sido hervidos en forma conjunta con otras plantas debido que de ser así sería de esperar que se produjeran niveles altos de ácido mirístico.

Si bien la evidencia faunística del sitio muestra una abundante cantidad de restos de peces, que avalarían el origen de los residuos de ácidos grasos a partir de este recurso, por el momento no se considera oportuno descartar la posibilidad de la utilización de algún vegetal típico del bosque de tala o de maíz como elemento de la dieta, aunque no se han recuperado restos macroscópicos.

\section{Consideraciones}

Los estudios sobre la relación forma-función aquí desarrollados han considerado a la tecnología como a un fenómeno cultural complejo embebido en una visión de mundo históricamente específica, con una acción social estratégica que involucra el factor humano a nivel individual y colectivo (Drobes y Hoffman 1994). Se considera a la tecnología conformada por los conocimientos y herramientas específicos del grupo que conjuntamente con las materias primas componen los bienes de producción, estos aspectos posibilitan la comprensión de los principios de organización del grupo mediante inferencias sobre el esfuerzo invertido, la destreza del artesano, la existencia o no de estandarización, la localización espacial y el contexto de producción (Costin 2000). Se han analizado algunos de los aspectos antes citados para explorar la dimensión social de la tecnología; se ha partido de la identificación de atributos técnicos, cadenas operativas y variabilidad morfo-funcional que constituyen el insumo para aproximarnos a estas consideraciones.

Se propone a través del análisis morfofuncional que las características morfo-tecnológicas de los contenedores de procesamiento responden a una especificidad para las actividades domésticas y en particular para la actividad culinaria.

Dentro del contexto de emergencia de la cerámica en América, Crown y Wills (1995) han argumentado que la composición de la pasta, los perfiles simples y las paredes delgadas de los recipientes que caracterizan la cerámica utilitaria temprana son todos rasgos apropiados para vasos usados para cocinar sobre fuego abierto. Si bien son características deseables en recipientes de cocina, se puede decir que tales atributos resultan ventajosos en general, y pueden reflejar una solución común a problemas asociados a la reducción del stress térmico tanto en la cocción de la pieza como en la actividad culinaria, más que vinculado a la función de la vasija. La conducta termal de una cerámica concierne dos situaciones: el horneado inicial de la arcilla y el uso de la vasija cocida por calentamiento. Así tales atributos no indican en sí mismos que la cocina fue la función primaria de estas vasijas (Heidke y HabichtMauche 1998; Heidke y Stark 1997).

En el presente trabajo, si bien se han tenido en cuenta las observaciones antes citadas, se han descrito a los contenedores de procesamiento con 
PALEO, M.C.; PEREZ MERONI, M. Dimensión social de la tecnología cerámica en sociedades cazadoras-recolectoras. Rev. do Museu de Arqueologia e Etnologia, São Paulo, 15-16: 73-85, 2005-2006.

formas esféricas, bocas amplias y bases cóncavas, presencia de superficies antideslizantes considerándolos óptimos para la cocción a fuego directo. También se han caracterizado sus pastas señalándose los atributos que tienden a minimizar el stress térmico. Por último se han evaluado las evidencias de uso y el análisis de los residuos orgánicos donde se plantea la posibilidad de la cocción de peces o vegetales en estos recipientes. Se considera que si bien los atributos aislados no indican que la cocina fue la función primaria de estos contenedores, la lectura conjunta de estos resultados permite sustentar su uso en la cocción y servicio y su especificidad morfo-tecnológica.

Se considera un aporte el trabajo con la variabilidad morfo-funcional de la muestra. La variabilidad en el conjunto alfarero se evidencia en la caracterización de tres tipos de contenedores procesamiento, almacenaje y transferencia - y que ha podido ser ampliada para los contenedores de procesamiento con la identificación de dos tipos de formas y la individualización de tres tamaños. Esto ha posibilitado proponer que estos grupos poseían un grado desarrollado de especialización en esta tecnología evidenciado por la presencia de vasijas con usos específicos que descartan la existencia de vasijas plurifuncionales, generalmente asociadas a una producción con un nivel no especializado.

Se interpreta que la existencia de determinantes funcionales en la selectividad de la pasta responde al manejo de recursos naturales del área y de sus propiedades.

El estudio de las pastas destaca la homogeneidad de las mismas. Atributos tales como la abundancia de feldespatos y la presencia de inclusiones intencionales de tiesto molido que otorgan a las piezas una buena respuesta al stress térmico, la presencia de pastas porosas acompañadas de un buen acabado de superficies internas y externas que imprimen a la pasta la cualidad de resistencia mecánica y de impermeabilidad, y la presencia de cuarzo que le otorga cuerpo a la estructura de la pieza permite proponer que estos grupos manejaban los recursos naturales del área y tal vez en función a conocimientos empíricos manipulaban sus propiedades. Así también al analizar los estudios aplicados en el centro este de Norte América (Meherer 1998) se observa que la adición de tiesto molido es considerada como un indicador de la relativa sofisticación de la tecnología cerámica, caracterización que se aplica al conjunto alfarero de Las Marías.
Se considera que como parte de las estrategias sociales utilizadas por estos grupos se puede postular un dominio más especializado de esta tecnología sustentado por la identificación de vasijas con usos específicos, experimentado conocimiento y manejo de recursos, cierto grado de sofisticación en la tecnología que expresaría la especialización de los artesanos. Con respecto a este punto el análisis de la manufactura y la decoración nos permitirían pensar en que varios individuos han sido los responsables del conjunto alfarero.

Al analizar las características del emplazamiento y los recursos potenciales planteados para el sitio Las Marías que comparte con los sitios de la región, se destaca la alta diversidad, abundancia y alternancia estacional de los recursos utilizados por estos grupos de ambientes ribereños. Esta caracterización estaría relacionada con situaciones de intensificación de los recursos evidenciada en cierta forma en los tipos de contenedores planteados donde el almacenamiento y procesamiento de los alimentos a través de la cocción es fundamental. Esta intensificación en los recursos acompañada de la especialización tecnológica propuesta permitiría sostener cierto grado de intensificación socio-económica. Es así que las características de la tecnología cerámica pueden considerarse como parte de las transformaciones culturales que se dan en el proceso de intensificación socio-económica.

Estas observaciones nos remiten a comentar el rol de la cerámica en sociedades cazadorasrecolectoras, en las cuales, se vincula al concepto de complejización social e intensificación económica. Diversos autores analizan el tema, algunos consideran que la cerámica entre cazadoresrecolectores es vista como una estrategia adaptativa causada por condiciones externas cambiantes, donde se daría una estrategia de movilidad reducida conduciendo a una intensificación económica y social. Así la intensificación estaría expresada en actividades relacionadas con el procesamiento de recursos alimenticios, donde la cerámica estaría involucrada en estas actividades (Oyuela Caicedo 1995). Otros destacan sus diferentes funciones como la de aumentar la comunicación e intercambio entre grupos productores y no productores, preparar y almacenar productos específicos para la subsistencia y contribuir al aumento en la diversificación de estrategias. Mencionan también la capacidad de 
explotar estacionalmente abundantes especies dado que aumenta las oportunidades para la preservación y almacenamiento, transporte y traspaso de alimentos (Hoopes, 1995).
Estas discusiones generalmente se instalan en contextos de emergencia de la cerámica, que si bien no es el caso aquí planteado, contribuye a comprender su dimensión social.

PALEO, M.C.; PEREZ MERONI, M. Social dimension of ceramic technology in hunter-gatherers societies. Rev. do Museu de Arqueologia e Etnologia, São Paulo, 15-16: 73-85, 2005-2006.

ABSTRACT: The objective of this work is to study in deph the morphological and functional variability of the ceramic assemblage from Las Marías site. The ceramic sample comes from a hunter-gatherer site from the late Holocene, placed in Magdalena department, Buenos Aires, Argentina. In a previous work, three categories of containers have been isolated: processing, storage and transference (Paleo and Pérez Meroni, in press). Processing containers will be particularly analysed through a methodology which has been developed to approach the relationship between shape and function, and which has allowed to understand the existing diversity. The studies of raw materials and traces of use, as well as the analysis of organic residues, have allowed us to infer about the range of activities in which the containers have been used. The research about the social dimension of ceramic technology has contributed to a better understanding of the different social strategies used by these groups.

UNITERMS: Ceramic technology - Methodology - Morpho-functional analysis Processing - Hunter-gatherers.

\section{Referencias bibliográficas}

BALESTA, B.; PALEO, M. C.; PÉREZ MERONI, M. M.; ZAGORODNY, N.

1997 Revisión y estado actual de las investigaciones arqueológicas en el Parque Costero Sur (Pdo. Magdalena; Prov. de Buenos Aires). M. Berón; G. Politis (Eds.) Arqueología pampeana en la década de los '90. Museo de Historia Natural de San Rafael. Mendoza. INCUAPA. FCS. UNCPBA: $147-160$.

BALFET, H.; FAUVET-BERTHELOT, M.F.; MOZON, S. 1992 Normas para la descripción de vasijas cerámicas. Centre D’études Mexicaines et Centramericaines. México.

CAVALLOTO, J. L.

1995 Evolución geomorfológica de la llanura costera en el margen sur del Río de la Plata. Tesis Doctoral inédita. Facultad de Ciencias Naturales y Museo. Universidad Nacional de CIGLIANO, E. M. la Plata, 237 páginas.

1963 Arqueología del Noreste de la provincia de Buenos Aires. Anales de la Comisión de Investigaciones Científicas de la Provincia de Buenos Aires. Vol IV: 471-511.
COSTIN, C.L.

2000 The use of ethnoarchaeology for the archaeological study of ceramic production. Journal of archaeological Method and Theory, 7 (4): 377-403.

CROWN, P.; WILLS, W. H.

1995 Economic intensification and the origins of ceramic containers in the American Southwest. W.K. Barnett; J.W. Hoopes (Eds.) The emergence of pottery: technology and innovation in Ancient Societies. Washington, D.C., Smithsonian Institution Press: 241254.

DE OLIVEIRACEZAR, F.

1895 Datos arqueológicos. Boletín del Instituto Geográfico Argentino. Buenos Aires, XVI: 264-271.

DOBRES, M. A.; HOFFMAN, C.

1994 Social Agency and Dynamics of Prehistoric Technology. Journal of Archaeological Method and Theory, 1 (3): 211-255.

FIDALGO, F.; COLADO, U.; DE FRANCESCO, F.

1973 Sobre ingresiones marinas en los partidos de Castelli, Chascomús y Magdalena (provincia 
PALEO, M.C.; PEREZ MERONI, M. Dimensión social de la tecnología cerámica en sociedades cazadoras-recolectoras. Rev. do Museu de Arqueologia e Etnologia, São Paulo, 15-16: 73-85, 2005-2006.

de Buenos Aires). Actas V Congreso Geológico Argentino, III: 103-138.

HEIDKE, J.; STARK, M.

1997 Ceramic container technology in the early saothwestern far ming village: inception, adoption, and intensification. J. Mabry (Ed.) Early Settlements in the Southern Southewes, in press.

HEIDKE, J.; HABICH-MAUCHE, J.

1998 The first occurrences and early distribution of pottery in the North American southewest. Revista de Arqueología Americana, 14, enero-junio. Instituto Panamericano de Geografía e Historia: 65-99.

HOOPES, J.

1995 Interaction in Hunting and Gathering Societies as Context for the emergence of pottery in the Central American Isthmus. W. Barnett, J. Hoopes (Eds.) The emergence of pottery. Washington and London, Smithsonian Institution Press: 185-198.

MALAINEY, M

1997 The reconstruction and Testing of Subsistence and Settlement strategies for the Plains, Parkland and Southern Boreal Forest. Dessertation University of Manitoba. Winnipeg. Canadá.

MEHERER, M.

1998 Early pottery in Midwestern North America. Arqueología Americana, 14: 135-151.

MORENO, F. P.

1874 Noticias. Sobre antiguedades de los Indios, del tiempo anterior á la conquista. Boletín de la Academia Nacional de Ciencias Exactas, I, Buenos Aires: 130 - 149.

ORTON, C.; TYERS, P.; VINCE, A.

1997 La cerámica en arqueología. Barcelona, España: Ed. Crítica.

OYUELACAICEDO,A.

1995 Rocas versus arcilla. La evolución de la tecnología cerámica en el caso de San Jacinto 1, Colombia. W. Barnett; J. Hoopes (Eds.) The emergence of Pottery. Technology and Innovation in Ancient Societies. Washington y Londres. Smithsonian Institution Press: 133-144.

PALEO, M. C.; PAEZ; M.; PEREZ MERONI, M. M.

2000 Condiciones ambientales y ocupación humana durante el Holoceno tardío en el litoral fluvial bonaerense. D. Mazzanti; M. Berón; F. Oliva (Eds.) Del mar a los salitrales. Facultad de Humanidades. Universidad Nacional de Mar del Plata. Argentina: 365-376.

PALEO, M.C.; PÉREZ MERONI, M.M.

1995 Análisis cerámico en grupos pescadorescazadores-recolectores del Litoral Bonaerense. Sitio El Ancla. Partido de Magdalena. Provincia de Buenos Aires. M. Consens; J.M.
López Mass; M.C. Curbelo (Eds.) Arqueología en el Uruguay: 398-405.

1999 Seguimiento de la cadena operativa en la manufactura cerámica. Contribuciones arqueológicas, 5. Museo Regional de Atacama. Chile. T.I: 299-309.

2004 Problemáticas vinculadas a las estrategias de subsistencia de la Localidad Arqueológica Barrio San Clemetne. C.J. Gradín; F. Oliva (Eds.) La Región Pampeana - su pasado arqueológico: 311-319.

En prensa (1) Primeros resultados del sitio "Las Marías” Partido de Magdalena Provincia de Buenos Aires. XIV Congreso Nacional de Arqueología Argentina, 2001. Facultad de Humanidades y Artes. Universidad Nacional de Rosario.

En prensa (2) Relación forma-función en un conjunto alfarero del Partido de Magdalena, provincia de Buenos Aires. Aproximación metodológica. Actas del XV Congreso Nacional de Arqueología Argentina, 2004. Río Cuarto. Córdoba.

PÉREZ MERONI, M. M.; BLASI, A.

1999 Sitio arqueológico “El Ancla”, provincia de Buenos Aires. Ensayo y experimentación de sedimentos pelíticos locales para la manufactura cerámica. M. Berón; G. Politis. (Eds.) Arqueología pampeana en la década de los '90. Museo de Historia Natural de San Rafael. Mendoza. INCUAPA. FCS. UNCPBA.

PÉREZ MERONI, M.; PALEO, M. C.

1999 La utilización del espacio por grupos del Litoral Bonaerense. J. M. López Mass; M. Sans (Comp.) Arqueología y bioantropología de las Tierras Bajas. Uruguay: 165-172.

POLITIS, G.

1988 Paradigmas, modelos y métodos en la arqueología de la Pampa bonaerense. $\mathrm{H}$. Yacobaccio (Ed.) Arqueología contemporánea argentina. Actualidad y perspectivas. Buenos Aires, Ediciones Búsqueda: 59-107.

POLITIS, G.; MADRID, P.

2001 Arqueología pampeana: estado actual y perspectivas. E. Berberían; A. Nielsen (Eds.) Historia Argentina Prehispánica II. Córdoba, Brujas: 737-814.

SHEPARD, A.

1976 Ceramics for the archaeologist. Carnegie Institution of Washington. Publication 609.

RICE, P.

1989 Pottery analysis. A sourcebook. Chicago: University of Chicago Press.

SINÓPOLI, C.

1991 Approaches to archaeological ceramics. New York and London: Plenum Press.

SKIBO, J.

1992 Pottery function: a use - alteration perspective. New York and London: Plenum Press. 